\title{
The relationship between high resolution computed tomography and pulmonary function tests in sarcoidosis
}

\author{
Nilüfer Aykaç (D) \\ Department of Chest Diseases, Gayrettepe Florence Nightingale Hospital, Istanbul, Turkey
}

\begin{abstract}
Objectives: This study aims to assess the correlation between inspiration and expiration high resolution computed tomography (HRCT) findings and pulmonary function tests (PFTs) in patients with sarcoidosis.

Patients and methods: Forty-five patients with sarcoidosis (18 males, 27 females; mean age $42.1 \pm 11.6$ years; range, 24 to 64 years) were included in this single-center, prospective, non-interventional study. Disease stage, presence and level of air trapping in expiratory and inspiratory HRCT patterns such as nodular, reticular, fibrotic and ground-glass patterns, and PFT results were recorded. The correlations between HRCT findings and PFTs were analyzed.

Results: Diagnoses of sarcoidosis were histopathologically (69\% cases) or radiologically confirmed. According to X-ray findings, $3(6.6 \%), 15$ ( $33.3 \%)$, $24(53.3 \%)$, and $3(6.6 \%)$ patients were categorized as stage $0, \mathrm{I}, \mathrm{II}$ and III sarcoidosis, respectively. Air trapping was detected in HRCT in 33 (73.3\%) patients. Although there was no association between air trapping and inspiratory imaging patterns and/or pulmonary function tests, they seemed more abundant in the lower/mid lung zones. Forced expiratory volume in one second (FEV1)/forced vital capacity (FVC) ratio and forced expiratory flow (FEF) 25-75 was significantly lower in patients with fibrosis. Significant negative correlations between the fibrotic pattern and FEV1/FVC $(r=-0.354$; $\mathrm{p}<0.005)$ and $\mathrm{FEF}_{25-75}(\mathrm{r}=-0.440 ; \mathrm{p}<0.005)$ values were detected.

Conclusion: Although air trapping pattern in expiratory HRCT was not associated with pulmonary function tests, it may be a useful diagnostic parameter in assessing the level of parenchymal involvement of the lungs in sarcoidosis. Among the imaging patterns on HRCT, only fibrosis was associated with airway obstruction.

Keywords: Expiratory high resolution computed tomography, inspiratory high resolution computed tomography, parenchymal fibrosis, sarcoidosis.
\end{abstract}

Sarcoidosis is an idiopathic granulomatous disease which affects multiple organ systems in the body, mostly the lungs. ${ }^{[1,2]}$ It can affect patients of any age, sex, or race, but most typically affects people younger than 40 years, with peak incidence in the third decade of life. ${ }^{[3]}$ The prevalence of sarcoidosis in the general population is estimated between 1-40 cases per 100,000 people. ${ }^{[4]}$

Although this disease was initially recognized over a century ago, there are still many uncertainties regarding its etiology, treatment, and prognosis. ${ }^{[5]}$ The diagnosis of sarcoidosis is also a matter of debate. ${ }^{[6,7]}$ In most cases, diagnosis is established through clinical and radiographic findings supported by histologic evidence of noncaseating granulomas. ${ }^{[7]}$ Other causes of such granulomatous inflammation must also be excluded. ${ }^{[2]}$

Over the past two decades, our understanding of sarcoidosis has evolved with the advancements in novel diagnostic tools. The development of highresolution computed tomography (HRCT) has resulted in improved imaging for the assessment of subtle parenchymal details, enabling discrimination between inflammation and fibrosis among patients with pulmonary sarcoidosis. ${ }^{[8-12]}$ In addition to enhanced diagnostic accuracy, HRCT images may be beneficial in planning its management and evaluating treatment outcomes. ${ }^{[13]}$ However, there

Received: June 09, 2020 Accepted: June 26, 2020 Published online: August 25, 2020

Correspondence: Nilüfer Aykaç, MD. Gayrettepe Florence Nightingale Hastanesi Göğüs Hastalıkları Bölümü, 34349 Beşiktaş, İstanbul, Türkiye. Tel: +90 212 - 2883400 e-mail: niluferkongar@gmail.com 
are conflicting reports regarding the correlation between HRCT findings and the results of pulmonary function test (PFT). ${ }^{[13-15]}$ Therefore, the purpose of this study was to assess the contribution of expiratory and inspiratory HRCT in the evaluation of the parenchymal changes in the lungs and to determine the correlation between HRCT and PFT findings in patients with sarcoidosis.

\section{PATIENTS AND METHODS}

\section{Study population}

A total of 45 patients (18 males, 27 females; mean age $42.1 \pm 11.6$ years; range, 24 to 64 years) with sarcoidosis treated at our institution were included in this single-center prospective non-interventional study. The histopathological diagnosis of sarcoidosis in clinically suspected patients required presence of noncaseating granulomatous inflammation in biopsy specimens. Criteria for clinicoradiographic diagnosis included patients who did not accept biopsies or whose performance status was not suitable for such an invasive procedure, presence of Lofgren's syndrome $e^{[2,16]}$ and/or exclusion of other granulomatous diseases, and lack of any other non-sarcoidosis disease within the course of six months was required. Patients were staged into four groups according to their chest x-ray findings as described by Scadding. ${ }^{[17]}$ Those with diseases which may cause mosaic attenuation pattern in the lungs (i.e. chronic pulmonary thromboembolism, primary pulmonary hypertension, cardiac diseases, obstructive sleep apnea syndrome, and neuromuscular diseases) and patients who did not have single-breath nitrogen elimination test were excluded from the study.

\section{High resolution computed tomography}

All patients underwent HRCT with $512 \times 512$ reconstruction matrix, $120 \mathrm{kVp}, 100 \mathrm{mAs}$ and 1.9 seconds scan time (Philips Tomoscan LX, Amsterdam, the Netherlands). High-resolution computed tomography images were obtained from the apex to the base of the lungs in supine position during forced inspiration with $1 \mathrm{~cm}$ section intervals and $1.5 \mathrm{~mm}$ slice thickness. Afterwards, the scans were repeated during expiration with $2 \mathrm{~cm}$ section intervals and $1.5 \mathrm{~mm}$ slice thickness. No contrast material was injected during HRCT. Standard window level/width parameters were set to 600-700/100-1500 Hounsfield unit (HU).

High-resolution computed tomography images were assessed separately by two radiologists who were blinded to the clinical information of the patients and presence of five typical radiographic features suggestive of sarcoidosis was determined as follows: ${ }^{12]}$

1. Air trapping (AT): focal areas of decreased attenuation in the lung parenchyma expiratory CT images

2. Nodular pattern: round and self-limited parenchymal opacities

3. Linear reticular pattern: linear or reticular opacities which are caused by interlobular and intralobular septal thickening

4. Ground-glass opacities: Areas with high attenuation where bronchus, vessels and airways can be seen; and

5. Fibrosis: Subpleural, well surrounded air cysts with 1-3 $\mathrm{mm}$ thickness (honeycomb-like cysts), architectural distortion (displacement of fissures and bronchovascular bundles) and traction bronchiectasis (expansion of bronchus and bronchioles due to elastic tension in the fibrosis areas.

Presence of more than one dominant feature was considered "mixed pattern". The correlation of the above-mentioned features with the disease stages and PFT findings were assessed.

Patients in whom air trapping was observed only in expiratory HRCT were classified as "Type A", whereas patients in whom air trapping was detected in both expiratory and inspiratory HRCTs were classified as "Type B".

\section{Air trapping score}

The level of parenchymal involvement of each lung zone was visually scored on a $0-5$ scale and mean AT score was calculated. The differences in AT scores among different lung zones were evaluated.

\section{Evaluation of the degree of radiographic findings in HRCT}

The patients were further categorized into five groups according to the degree of radiographic features observed in HRCT: 0: 
No evidence of disease; 1: 1-25\% involvement of lung parenchyma; 2: 26-50\% involvement of lung parenchyma; 3. 51-75\% involvement of lung parenchyma; and 4: 76-100\% involvement of lung parenchyma.

\section{Pulmonary function tests}

The spirometric assessments (Sensor Medics Vmax 2130 V6200 6200) of the patients were performed on the same day as HRCT and the values were calculated as the percent of the normal values according to age, sex, and height of the patient. Diffusion capacity of the lung for carbon monoxide (DLCO), DLCO/alveolar ventilation (VA) ratio, total lung capacity (TLC), and residual volume (RV) were measured with single-breath nitrogen elimination test. Forced expiratory volume in one second (FEV1)/forced vital capacity (FVC) ratio $<70 \%$, was accepted as obstructive pattern, FVC $<80 \%$ as restrictive pattern, and RV/TLC $>40 \%$ as hyperinflation. A forced expiratory flow (FEF) 25-75 lower than the $60 \%$ of the expected value was defined as decreased maximal mid-expiration flow (MMF). Diffusion capacity of the lung for carbon monoxide value lower than $81 \%$ of the expected value was considered decreased.

The correlation between the stage of the disease, the most commonly observed HRCT pattern, and AT score was investigated. Moreover, the association between inspiratory HRCT patterns, presence and score of TA, and PFT parameters (i.e. FEV1, FVC, FEV1/FVC, FEF25-75, TLC, RV, RV/TLC, DLCO, DLCO/VA) was determined.

\section{Statistical analysis}

Statistical package for social science (SPSS) for Windows version 16.0 (Chicago, Illinois, United States of America) was used for statistical analyses. Student's t-test or Mann-Whitney $\mathrm{U}$ tests were applied for numerical variables, whereas chi-square, McNemar, or Fisher's exact tests were utilized for comparing the percentage of groups, when applicable. The correlation between numerical variables was evaluated with Spearman's correlation test. Statistical significance level was set as $p<0.05$.

\section{Ethics}

The study was performed in accordance with the 2000 Declaration of Helsinki and was approved by the Ethics Committee of the Medeniyet University Goztepe Training and Research Hospital, Istanbul. All patients provided their informed consent prior to their inclusion in the study.

\section{RESULTS}

\section{General characteristics of the patients}

The diagnosis of sarcoidosis was confirmed histopathologically in most cases $(\mathrm{n}=31 ; 69 \%)$ cases, via mediastinoscopy $(\mathrm{n}=11)$, transbronchial or open lung biopsies ( $\mathrm{n}=8$ and $\mathrm{n}=2$ ), or via either bronchial mucosa, dermal, lymph node or lip biopsies; and otherwise based on radiological findings. According to x-ray findings, 3 (6.6\%), 15 (33.3\%), 24 (53.3\%), and $3(6.6 \%)$ patients were categorized as stage 0 , I, II, and III sarcoidosis, respectively. Of the patients, 38 (84.4\%) were non-smokers. Corticosteroid and hydroxychloroquine treatments were being administered to 5 (11.1\%) and 4 (8.8\%) patients, respectively.

\section{Inspiratory high-resolution computed tomography patterns}

Inspiratory HRCT images of 18 (40\%) patients were normal; 15 patients had nodular, reticular or fibrosis patterns, while others $(n=12)$ had a mixed pattern (Table 1).

Table 1. Distribution of the patterns observed in inspiratory HRCT $(n=27)$

\begin{tabular}{lc}
\hline Inspiratory HRCT patterns & $\mathrm{n}$ \\
\hline Nodular & 10 \\
Reticular & 2 \\
Fibrosis & 3 \\
Mixed & 12 \\
Nodular + reticular & 1 \\
Nodular + fibrosis & 1 \\
Reticular + fibrosis & 3 \\
Fibrosis + ground-glass* & 1 \\
Nodular + ground-glass + fibrosis & 1 \\
Nodular + reticular + fibrosis & 3 \\
Nodular + ground-glass + reticular & 1 \\
Nodular + ground-glass + reticular + fibrosis & 1 \\
\hline
\end{tabular}

HRCT: High-resolution computed tomography; * None of the patients had this pattern solely. 
Table 2. Comparison of the zones of the lung according to presence of AT

\begin{tabular}{|c|c|c|c|c|}
\hline \multirow[b]{2}{*}{ Zones of the lung } & \multicolumn{2}{|c|}{ Upper zone* } & \multicolumn{2}{|c|}{ Lower zonet } \\
\hline & AT $(-)$ & AT $(+)$ & AT $(-)$ & AT $(+)$ \\
\hline \multicolumn{5}{|l|}{ Mid zone ${ }^{*} \dagger$} \\
\hline AT $(-)$ & 16 & 1 & 13 & 4 \\
\hline AT $(+)$ & 8 & 20 & 3 & 28 \\
\hline \multicolumn{5}{|l|}{ Lower zone* } \\
\hline AT (-) & 14 & 2 & & \\
\hline AT $(+)$ & 10 & 19 & & \\
\hline
\end{tabular}

Table 3. Pulmonary function test results of the patients (\% of the estimated values)

\begin{tabular}{lcc}
\hline & Mean \pm SD & Range \\
\hline FVC & $89.5 \pm 21.3$ & $36-150$ \\
FEV1 & $96.7 \pm 19.4$ & $46-155$ \\
FEV1/FVC & $77.9 \pm 8.4$ & $49-96$ \\
FEF $25-75_{\text {RV }}$ & $69.2 \pm 4.7$ & $14-138$ \\
TLC & $109.4 \pm 46.5$ & $42.6-263.7$ \\
RV/TLC & $98.0 \pm 18.2$ & $62.5-143.2$ \\
DLCO/VA & $108.1 \pm 32.2$ & $52.6-188$ \\
\hline
\end{tabular}

SD: Standard deviation; FVC: Forced vital capacity; FEV1: Forced expiratory volume in one second; FEF: Forced expiratory flow; RV: Residual volume; TLC: Total lung capacity; DLCO: Diffusion capacity of the lung for carbon monoxide; VA: Alveolar gas volume.

Table 4. Correlation of HRCT patterns and AT scores according to disease stage

\begin{tabular}{lccccc}
\hline & & \multicolumn{3}{c}{ Inspiratory HRCT pattern } \\
\cline { 3 - 6 } Disease state vs. & AT score & Nodular & Ground-glass & Reticular & Fibrosis \\
\hline $\mathrm{r}$ & 0.236 & 0.134 & 0.160 & 0.173 & 0.459 \\
$\mathrm{P}$ value & 0.499 & 0.172 & 0.640 & 0.482 & 0.006 \\
\hline
\end{tabular}

HRCT: High-resolution computed tomography; AT: Air trapping; r: Spearman's correlation coefficient.

\section{Air trapping}

In 29 patients, AT was seen on expiratory HRCT only (Type A), while four patients were AT (+) on both inspiratory and expiratory HRCT (Type B). No pathological findings were detected on inspiratory HRCT in 18 patients, of which 11 had AT on expiratory HRCT. Air trapping score was $8.1 \pm 13.0$ (range: 0-65). Air trapping images were more abundant in the lower/mid zones of the lungs, compared to the upper zones $(p=0.039)$ (Table 2).

\section{Pulmonary function test results}

Individual assessment of PFTs revealed obstruction, restriction, mixed, and small airway obstruction in 6, 4, 1, and 11 patients, respectively; and 15 patients had decreased DLCO (Table 3).

Relationship between disease stages, HRCT patterns and air trapping

When the relationship between HRCT patterns and disease stage were evaluated, presence of only fibrosis was found to be positively correlated 
Table 5. The association between inspiratory HRCT patterns and AT scores $(n=45)$

\begin{tabular}{lccc} 
& \multicolumn{2}{c}{ AT score } & \\
\cline { 2 - 3 } & $\mathrm{n}$ & Mean $\pm \mathrm{SD}$ & $p$ \\
\hline Nodular & 18 & $9.2 \pm 15.1$ & 0.803 \\
Non-nodular & 27 & $8.1 \pm 13.9$ & \\
Reticular & 11 & $7.0 \pm 7.5$ & 0.576 \\
Non-reticular & 34 & $9.0 \pm 15.9$ & \\
Ground-glass & 4 & $19.8 \pm 30.3$ & \multirow{2}{*}{0.476} \\
Non-ground-glass & 41 & $7.4 \pm 11.7$ & \\
Fibrosis & 13 & $12.8 \pm 23.1$ & 0.369 \\
Non-fibrosis & 32 & $6.7 \pm 8.1$ & \\
\hline
\end{tabular}

HRCT: High-resolution computed tomography; AT: Air trapping; SD: Standard deviation.

with the stage of the disease $(\mathrm{r}=0.459 ; \mathrm{p}=0.006)$ (Table 4). On the other hand, no relationship was detected between HRCT patterns and AT scores (Table 5).

\section{Relationship between HRCT and pulmonary} function tests

When the patients were classified in four groups according to the presence of HRCT patterns and their PFT results were compared, the patients with fibrosis pattern demonstrated significantly lower FEV1/FVC $(p=0.029)$ and $\mathrm{FEF}_{25-75 \quad(p=0.005)}$ values, compared to the patients without fibrosis (Table 6). When the correlation between the degree of the four HRCT patterns and PFT parameters were analyzed, the fibrosis-only pattern was found to be negatively correlated with FEV1/FVC and FEF25-75 values (Table 7).

\section{Relationship between pulmonary functions and air trapping}

Expiratory HRCT of five of the six patients who had obstruction on PFT demonstrated AT. Air trapping score was $17.5 \pm 22.9$ and $6.7 \pm 11.5$ in patients with FEV1/FVC $<70 \%$ and FEV1/FVC $\geq 70 \%$, respectively. Although mean AT score was higher among patients with FEV1/FVC $<\% 70$, this difference did not reach the level of statistical significance $(p=0.100)$. Similarly, AT score was $16.5 \pm 23.6$ and $5.2 \pm 6.4$ in patients with $\mathrm{RV} / \mathrm{TLC} \geq 40 \%$ and $\mathrm{RV} / \mathrm{TLC}$ $<40 \%$, respectively; with a difference that was not statistically significant $(p=0.200)$. AT was detected in one of the four patients

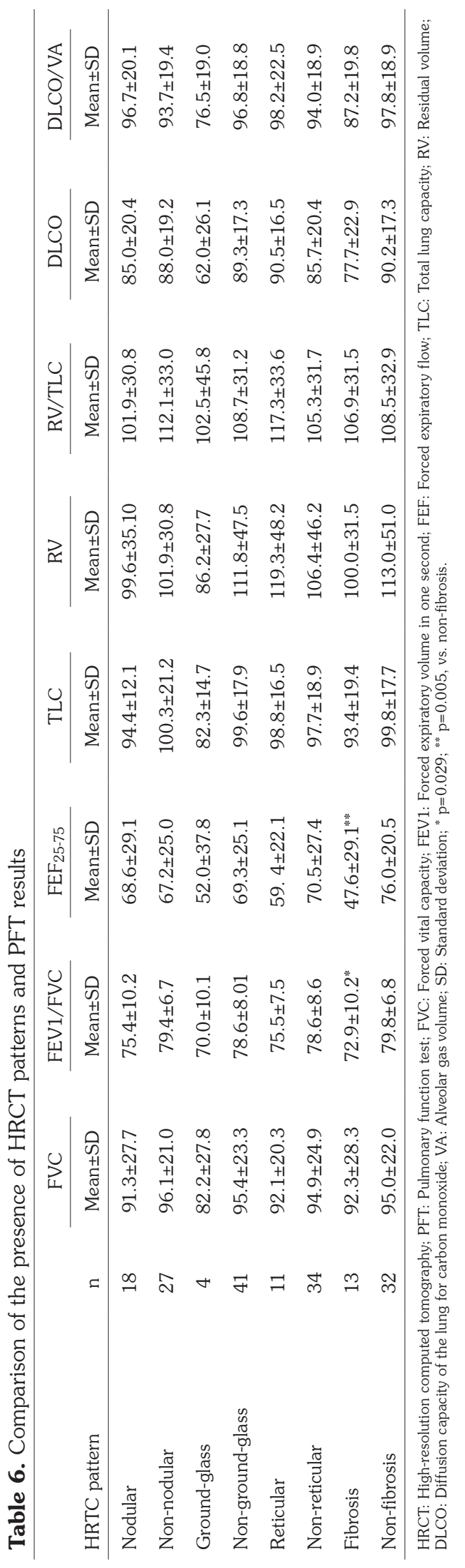


Table 7. The correlation between the degree of HRCT patterns and PFT parameters

\begin{tabular}{|c|c|c|c|c|}
\hline \multirow[b]{3}{*}{ PFT } & \multicolumn{4}{|c|}{ HRCT pattern } \\
\hline & \multicolumn{2}{|c|}{ Correlation } & \multicolumn{2}{|c|}{ Coefficient $(r)$} \\
\hline & Nodular & Ground-glass & Reticular & Fibrosis \\
\hline FVC & 0.089 & -0.15 & -0.08 & 0.60 \\
\hline FEV1 & 0.017 & -0.211 & -0.149 & -0.257 \\
\hline FEV1/FVC & -0.257 & -0.286 & -0.198 & $-0.354^{*}$ \\
\hline $\mathrm{FEF}_{25-75}$ & -0.001 & -0.102 & -0.239 & $-0.440^{*}$ \\
\hline TLC & -0.72 & -0.284 & 0.71 & -0.165 \\
\hline $\mathrm{RV}$ & -0.163 & -0.181 & 0.120 & -0.21 \\
\hline RV/TLC & 0.215 & -0.155 & 0.155 & 0.04 \\
\hline DLCO & -0.153 & -0.29 & 0.10 & -0.257 \\
\hline DLCO/VA & 0.046 & 0.283 & 0.062 & -0.232 \\
\hline
\end{tabular}

HRCT: High-resolution computed tomography; PFT: Pulmonary function test; FVC: Forced vital capacity; FEV1: Forced expiratory volume in one second; FEF: Forced expiratory flow; TLC: Total lung capacity; RV: Residual volume; DLCO: Diffusion capacity of the lung for carbon monoxide; VA: Alveolar gas volume; ${ }^{*} \mathrm{p}<0.005$.

with restriction, one patient with mixed type respiratory function disorder, and in eight of the twelve patients with small airway obstruction. Of the 32 patients who had normal PFT results, 25 had AT. There was no statistically significant difference between the PFT parameters of the patients with and without AT on expiratory HRCT, and no statistically significant correlation between any of the PFT parameters and AT score on the expiratory HRCT (Table 8).

\section{DISCUSSION}

The results of our study indicated that air trapping patterns in expiratory HRCT were not associated with pulmonary function tests, but may be a useful diagnostic parameter in assessing the level of parenchymal involvement

Table 8. The correlation coefficients between pulmonary function test parameters and air trapping scores

\begin{tabular}{lc}
\hline & \multicolumn{1}{c}{$\mathrm{r}^{*}$} \\
\hline FVC & -0.039 \\
FEV1 & -0.083 \\
FEV1/FVC & -0.082 \\
FEF $25-75$ & -0.167 \\
RV & 0.131 \\
RV/TLC & 0.181 \\
DLCO & -0.006 \\
\hline
\end{tabular}

FVC: Forced vital capacity; FEV1: Forced expiratory volume in one second; FEF: Forced expiratory flow; RV: Residual volume; TLC: Total lung capacity; DLCO Diffusion capacity of the lung for carbon monoxide; * None was significant. of the lungs in sarcoidosis. In addition, it was observed that, among the imaging patterns on HRCT, only fibrosis was associated with airway obstruction.

High-resolution computed tomography is considered to be the most sensitive radiological technique, transbronchial lung biopsy may be required in cases in which $\mathrm{HRCT}$ findings are inconclusive..$^{[9,10,18]}$ AT can be detected with expiratory HRCT among patients with minimal or no changes in inspiratory HRCT. ${ }^{119-21]}$ In the present study, AT could be detected with expiratory HRCT in 11 patients with no AT image on inspiratory HRCT, confirming the previously published outcomes. ${ }^{[22]}$ Davies et al. ${ }^{[18]}$ evaluated the radiologic images of 21 patients with sarcoidosis and reported that all 20 of the patients with AT appearance also had other parenchymal lesions. Our findings contradict with those of Davies et al.'s ${ }^{[18]}$ study, as we have demonstrated that AT image can be the only sign of parenchymal involvement in patients with sarcoidosis. Therefore, we suggest that expiratory HRCT may provide additional benefit in diagnosing sarcoidosis among patients with negative inspiratory HRCT findings. AT develops due to airway obstruction or decreased pulmonary compliance in sarcoidosis. ${ }^{[20]}$ In the present study, 33 (73.3\%) of 45 patients had AT on expiratory HRCT. Previous studies reported this percentage as 83-95\%. ${ }^{[22]}$ Magkanas et al. ${ }^{[22]}$ evaluated the correlation of expiratory 
HRCT findings with inspiratory patterns and pulmonary function tests, and reported that $83 \%$ of the 30 patients had AT on expiratory HRCT, whereas only $16 \%$ had this appearance on inspiratory HRCT. Similarly, we detected AT in $87.9 \%$ of the patients during expiration imaging and only $12.1 \%$ of the patients had this image on both inspiratory and expiratory HRCT. Although it has been well established that sarcoidosis more frequently affects upper and middle zones, AT appearance are not commonly detected in those zones. Magkanas et al. ${ }^{[22]}$ reported that AT was more common in the lower zones; however, this difference did not reach a statistically significant level, probably due to the low sample size. Similarly, we detected that AT appearance was more common in the middle and lower zones, and this difference was statistically significant. It has been proposed that wide-spread localization of the granulomas to the lower lobes of the lungs can be indirectly visualized as AT appearance. ${ }^{[22]}$

We also determined the HRCT patterns which can be associated with AT appearance, however we could not identify any difference between different HRCT patterns according to AT zone. Magkanas et al. ${ }^{[22]}$ reached the same conclusion, as they were only able demonstrate a weak correlation between fibrotic pattern and AT score. ${ }^{[22]}$ In our series, we could not detect any significant correlation between the stage of the disease and AT score. On the other hand, there was a positive correlation between fibrosis pattern and stage of the disease. This phenomenon can be explained by the enhanced fibrosis at the advanced stages of the disease. Recently, one of the most commonly searched topics is the association between AT images on expiratory HRCT and PFT. Hansell et al. ${ }^{[23]}$ detected AT in 40 (89\%) of 45 patients and found a significant correlation between AT and small airway obstruction. Davies et al. ${ }^{[18]}$ found significant correlation between AT score and both RV/TLC and FEF 25-75. ${ }^{[23]}$ Similarly, Magkanas et al. ${ }^{[22]}$ demonstrated a significant correlation between AT and both RV and RV/TLC. However, we could not detect any correlation between AT and PFT parameters.

Studies evaluating the correlation between the radiologic characteristics of sarcoidosis and PFT parameters report conflicting results. ${ }^{[20]}$ Levinson et al. ${ }^{[24]}$ compared chest x-ray findings according to PFT in 18 patients and found PFT deterioration without any changes in chest $\mathrm{X}$-ray. Austin ${ }^{[25]}$ compared chest x-ray findings with that of CT and concluded that CT is a superior technique in demonstrating clinical and functional deterioration. On the other hand, in their study, Müller et al. ${ }^{[26]}$ failed to demonstrate the superiority of CT over chest $\mathrm{X}$-ray in terms of assessing functional problems. Despite these conflicting results, most authors agreed on the advantages of CT over chest $\mathrm{X}$-ray in demonstrating the presence, extent, and distribution of parenchymal involvement in patients with sarcoidosis. However, they also admitted that CT findings play a limited role in predicting pulmonary function outcomes. The association between inspiratory HRCT patterns and PFT parameters has been evaluated in sarcoidosis patients by numerous studies. Due to the utility of HRCT in the evaluation of diffuse interstitial lung diseases, various patterns have been analyzed. However, there are conflicting results regarding the correlation between these CT patterns and PFT. ${ }^{[27]}$ In 1989 Müller et al. ${ }^{[10]}$ first assessed the correlation between CT patterns and clinical, functional, and radiological findings in 27 sarcoidosis patients. The authors demonstrated that the degree of the disease in CT is correlated with dyspnea, TLC, and DLCO. They also noted that patients with reticular opacities were more likely to suffer from dyspnea and had lower DLCO, compared to patients with nodular lesions. In another study, Remy-Jardin et al. ${ }^{[27]}$ analyzed the association between CT parameters and disease activity, functional changes, and prognosis. These authors could not find any correlation between the nodular pattern and PFT results, whereas they reported a weak but significant correlation with other CT patterns (consolidation, ground-glass opacity, and pulmonary distortion). However, Davies et al. ${ }^{[18]}$ failed to demonstrate any correlation between HRCT patterns and PFT. Abehsera et al. ${ }^{[1]}$ reported that sarcoidosis cases with fibrotic pattern who also have honeycomb appearance had lower TLC, VC, and DLCO values, whereas the ones with bronchial distortion had lower FEV1 and FEV1/FVC. Similarly, we found significantly lower $\mathrm{FEV} 1 / \mathrm{FVC}$ and $\mathrm{FEF}_{25-75}$ values among sarcoidosis patients in our study with fibrotic pattern compared to those without such a finding. Moreover, there was a significant negative 
correlation between the degree of the HRCT patterns and FEV1/FVC and $\mathrm{FEF}_{25-75}$ values. Hansell et al. ${ }^{[23]}$ found a significant association between reticular pattern and airway obstruction. The authors defined reticular pattern as images which include honeycomb lung and interseptal thickening, and concluded that this pattern indicated fibrotic lung. In our study, we could not demonstrate any significant correlation between reticular pattern and PFT parameters. On the other hand, our findings support those of Hansell et al. ${ }^{[23]}$ as we defined interseptal thickening as a component of reticular pattern, while honeycomb appearance was included in fibrotic pattern.

Noncaseating granulomas of sarcoidosis become detectable on HRCT images and form a characteristic appearance when they build up a conglomerated structure. Although it is suggested that these granulomas result in restrictive functional outcomes in most cases, there are also debates whether or not they may cause airway obstruction in sarcoidosis cases. ${ }^{[23,28-30]}$ It has been demonstrated that $70 \%$ of chronic sarcoidosis cases with fibrosis had airway obstruction and granulomas and/or peribronchial fibrosis causing airway narrowing and distortion proposed as the obstructing mechanisms. ${ }^{[31]}$ Abehsera et al..$^{[11]}$ also suggested that bronchial distortion can be responsible for airway obstruction. Similarly, obstruction among the patients with sarcoidosis who had fibrotic pattern in HRCT images in our study may be due to the destruction of the bronchial structure and compression caused by parenchymal fibrotic changes.

Our findings suggested that expiratory HRCT is an imaging modality which can provide additional benefit in the assessment of pulmonary parenchymal involvement for sarcoidosis patients who have normal inspiratory HRCT findings. However, an association between AT score and PFT parameters could not be demonstrated. Among all HRCT patterns, only the fibrosis pattern was negatively correlated with FEV1/FVC and $\mathrm{FEF}_{25-75}$, indicating airway obstruction.

\section{Acknowledgement}

We would like to thank Günay Can for the statistical analysis of the study and to Dr. Kemal Tahaoğlu, Tülin Sevim, Dr. Salih Güran and Dr. Güliz Ataç for their support in interpreting some of the data.

\section{Declaration of conflicting interests}

The author declared no conflicts of interest with respect to the authorship and/or publication of this article.

\section{Funding}

The author received no financial support for the research and/or authorship of this article.

\section{REFERENCES}

1. Baughman RP, Nagai S, Balter M, Costabel U, Drent $\mathrm{M}$, du Bois R, et al. Defining the clinical outcome status (COS) in sarcoidosis: results of WASOG Task Force. Sarcoidosis Vasc Diffuse Lung Dis 2011;28:56-64.

2. Hunninghake GW, Costabel U, Ando M, Baughman $\mathrm{R}$, Cordier JF, du Bois R, et al. ATS/ERS/ WASOG statement on sarcoidosis. American Thoracic Society/European Respiratory Society/ World Association of Sarcoidosis and other Granulomatous Disorders. Sarcoidosis Vasc Diffuse Lung Dis 1999;16:149-73.

3. Hillerdal G, Nöu E, Osterman K, Schmekel B. Sarcoidosis: epidemiology and prognosis. A 15-year European study. Am Rev Respir Dis 1984;130:29-32.

4. Henke CE, Henke G, Elveback LR, Beard CM, Ballard DJ, Kurland LT. The epidemiology of sarcoidosis in Rochester, Minnesota: a populationbased study of incidence and survival. Am J Epidemiol 1986;123:840-5.

5. Judson MA. Sarcoidosis: clinical presentation, diagnosis, and approach to treatment. Am J Med Sci 2008;335:26-33.

6. Judson MA. The diagnosis of sarcoidosis. Clin Chest Med 2008;29:415-27.

7. Baughman RP, Culver DA, Judson MA. A concise review of pulmonary sarcoidosis. Am J Respir Crit Care Med 2011;183:573-81.

8. Nishimura K, Itoh H, Kitaichi M, Nagai S, Izumi T. CT and pathological correlation of pulmonary sarcoidosis. Semin Ultrasound CT MR 1995;16:361-70.

9. Brauner MW, Grenier P, Mompoint D, Lenoir S, de Crémoux H. Pulmonary sarcoidosis: evaluation with high-resolution CT. Radiology 1989;172:467-71.

10. Müller NL, Kullnig P, Miller RR. The CT findings of pulmonary sarcoidosis: analysis of 25 patients. AJR Am J Roentgenol 1989;152:1179-82.

11. Abehsera M, Valeyre D, Grenier P, Jaillet H, Battesti JP, Brauner MW. Sarcoidosis with pulmonary fibrosis: CT patterns and correlation with pulmonary function. AJR Am J Roentgenol 2000;174:1751-7.

12. Criado E, Sánchez M, Ramírez J, Arguis $P$, de Caralt TM, Perea RJ, et al. Pulmonary sarcoidosis: typical and atypical manifestations at high-resolution CT with pathologic correlation. Radiographics 2010;30:1567-86. 
13. Gafà G, Sverzellati N, Bonati E, Chetta A, Franco F, Rabaiotti $\mathrm{E}$, et al. Follow-up in pulmonary sarcoidosis: comparison between HRCT and pulmonary function tests. Radiol Med 2012;117:968-78.

14. Murdoch J, Müller NL. Pulmonary sarcoidosis: changes on follow-up CT examination. AJR Am J Roentgenol 1992;159:473-7.

15. Akira M, Kozuka T, Inoue Y, Sakatani M. Longterm follow-up CT scan evaluation in patients with pulmonary sarcoidosis. Chest 2005;127:185-91.

16. Mañá J, Marcoval J. Skin manifestations of sarcoidosis. Presse Med 2012;41:e355-74.

17. Scadding JG. Prognosis of intrathoracic sarcoidosis in England. A review of 136 cases after five years' observation. Br Med J 1961;2:1165-72.

18. Davies CW, Tasker AD, Padley SP, Davies RJ, Gleeson FV. Air trapping in sarcoidosis on computed tomography: correlation with lung function. Clin Radiol 2000;55:217-21.

19. Arakawa H, Niimi H, Kurihara Y, Nakajima Y, Webb WR. Expiratory high-resolution CT: diagnostic value in diffuse lung diseases. AJR Am J Roentgenol 2000;175:1537-43.

20. Webb RW, Müller LN, Naidich DP. High-resolution CT of the lung. 3rd ed. Philadelphia: Lippincott Willams \& Wilkins; 2001.

21. Traill ZC, Maskell GF, Gleeson FV. High-resolution CT findings of pulmonary sarcoidosis. AJR Am J Roentgenol 1997;168:1557-60.

22. Magkanas E, Voloudaki A, Bouros D, Prassopoulos $\mathrm{P}$, Alexopoulou C, Tzanakis $\mathrm{N}$, et al. Pulmonary sarcoidosis. Correlation of expiratory highresolution $\mathrm{CT}$ findings with inspiratory patterns and pulmonary function tests. Acta Radiol 2001;42:494-501.

23. Hansell DM, Milne DG, Wilsher ML, Wells AU. Pulmonary sarcoidosis: morphologic associations of airflow obstruction at thin-section CT. Radiology 1998;209:697-704.

24. Levinson RS, Metzger LF, Stanley NN, Kelsen SG, Altose MD, Cherniack NS, et al. Airway function in sarcoidosis. Am J Med 1977;62:51-9.

25. Austin JH. Pulmonary sarcoidosis: what are we learning from CT? Radiology 1989;171:603-4.

26. Müller NL, Mawson JB, Mathieson JR, Abboud R, Ostrow DN, Champion P. Sarcoidosis: correlation of extent of disease at CT with clinical, functional, and radiographic findings. Radiology 1989;171:613-8.

27. Remy-Jardin M, Giraud F, Remy J, Wattinne L, Wallaert B, Duhamel A. Pulmonary sarcoidosis: role of CT in the evaluation of disease activity and functional impairment and in prognosis assessment. Radiology 1994;191:675-80.

28. Gleeson FV, Traill ZC, Hansell DM. Evidence of expiratory CT scans of small-airway obstruction in sarcoidosis. AJR Am J Roentgenol 1996;166:1052-4.

29. Shaw RJ, Djukanovic R, Tashkin DP, Millar AB, du Bois RM, Orr PA. The role of small airways in lung disease. Respir Med 2002;96:67-80.

30. Arakawa H, Webb WR. Expiratory high-resolution CT scan. Radiol Clin North Am 1998;36:189-209.

31. Miller A, Teirstein AS, Jackler I, Chuang M, Siltzbach LE. Airway function in chronic pulmonary sarcoidosis with fibrosis. Am Rev Respir Dis 1974;109:179-89. 